\title{
Estudio epidemiológico de la dermatitis atópica desde la farmacia comunitaria: Estudio DAFAC
}

\author{
Paqui Moreno \\ Farmacéutica comunitaria en Barcelona. Coordinadora del grupo de Dermatología de SEFAC.
}

\section{PALABRAS CLAVE}

dermatitis atópica, protocolo de actuación, criterios de derivación, dermatólogo, farmacéutico comunitario

\section{ABREVIATURAS}

DA: dermatitis atópica. DAFAC: Dermatitis Atópica en Farmacia Comunitaria.

\section{SEFAC: Sociedad}

Española de Farmacia Comunitaria.

\section{KEYWORDS}

atopic

dermatitis, interventional protocol, referral criteria, dermatologist, community pharmacist

\section{RESUMEN}

Objetivos: Diseñar y evaluar la utilidad de un protocolo de actuación del farmacéutico comunitario ante las consultas realizadas sobre dermatitis atópica en la farmacia comunitaria, acordando con dermatólogos los criterios de derivación. Conocer los motivos de consulta más habituales y determinar en qué casos constituyen motivo de derivación al médico.

Métodos: Estudio epidemiológico realizado en farmacias comunitarias españolas entre febrero del 2011 y enero del 2012. Se incluyeron en el estudio todos los usuarios de las farmacias que consultan un problema de salud dermatológico. Los farmacéuticos participantes, tras recibir formación específica online, aplicaron el protocolo de actuación consensuado.

Se registraron los motivos de consulta, los casos de derivación al médico, la existencia de sobreinfecciones, el uso de productos adecuados para el cuidado de la piel.

Resultados/Discusión: Participaron 259 farmacéuticos de 15 comunidades autónomas que registraron 688 intervenciones válidas. La distribución por sexo de los pacientes corresponde a 354 $(51,5 \%)$ de sexo masculino y $334(48,5 \%)$ del femenino, con mayor incidencia de la patología en menores de 14 años. En el 70\% de los casos no se requirió la derivación al dermatólogo. Motivos de consulta: prurito, irritación, dificultad para dormir, ansiedad y estrés, y sobreinfección (35\%), principal causa de derivación.

La elaboración y difusión del protocolo y criterios de derivación, así como los resultados del estudio a los socios de SEFAC (Sociedad Española de Farmacia Comunitaria) y al resto de farmacéuticos comunitarios permitirán la resolución de muchos de los casos de dermatitis atópica, contribuyendo así a la mejora de la calidad de vida del paciente.

Epidemiological study of atopic dermatitis from the community pharmacy perspective. The DAFAC study

\section{ABSTRACT}

Objectives: To design and evaluate the usefulness of a community pharmaceutical intervention in reference to atopic dermatitis consultations in the community pharmacy office, establishing consensus with dermatologists regarding the criteria for patient referral. To identify the most common reasons for consultation and determine which cases require referral to the physician.

Methods: An epidemiological study was carried out in Spanish community pharmacies between February 2011 and January 2012, including all pharmacy users consulting due to skin problems. After receiving specific online training, the participating pharmacists applied the consensus-based intervention protocol. The reasons for consultation were registered, along with the cases of referral to the physician, the existence of overinfections, and the use of adequate products for skin care.

Results/Discussion: A total of 259 pharmacies from 15 Spanish autonomous communities participated in the study, with the registry of 688 valid interventions. A total of 354 patients $(51.5 \%)$ were males and 334 (48.5\%) females, and disease conditions were found to be more frequent in patients under 14 years of age. In $70 \%$ of the cases referral to the dermatologist was not necessary. Reasons for consultation: itching, irritation, sleep disturbances, anxiety and stress, and overinfection $(35 \%)$ as the main cause of referral.

Development and diffusion of the protocol and referral criteria, as well as of the results of the study among the associates of SEFAC and the rest of community pharmacists will allow the resolution of many cases of atopic dermatitis - thereby contributing to improve patient quality of life.

Parte del contenido fue presentado en diversas comunicaciones en los Congresos Nacionales de Farmacéuticos Comunitarios de Valencia 2010 y Barcelona 2012 .

Recibido: 6-9-2013

Aceptado: 21-12-2013

Disponible online: 1-3-2014
Financiación: La plataforma online de formación Letiderma fue financiada por Laboratorios LETI. Conflicto de intereses: La autora declara no existir ningún conflicto de intereses en relación con el contenido del presente artículo. Cite este artículo como: Moreno P. Estudio epidemiológico de la dermatitis atópica desde la farmacia comunitaria: Estudio DAFAC. Farmacéuticos Comunitarios. 2014 Mar 01;6(1):12-17. doi:10.5672/FC.2173-9218.(2014/Vol6).001.03 


\section{Introducción}

La dermatitis atópica (DA) es una enfermedad inflamatoria cutánea crónica, con una alta predisposición genética y que se presenta en episodios de brotes. Incide principalmente en edades infantiles, hasta la pubertad, que es cuando mejora el cuadro en la mayoría de los casos (1-3). Se trata de un proceso multifactorial (2), en el cual desempeñan un papel diferentes factores como son:

- Predisposición genética.

- Alteraciones inmunológicas (4).

- Alteraciones vasculares.

- Alteraciones de la función de la barrera cutánea.

Un porcentaje muy elevado de pacientes atópicos tiene antecedentes familiares con alguna de las patologías que se incluyen en la denominada tríada atópica. En este sentido, muchos de los pacientes que en la edad infantil presentan dermatitis atópica, después, en la edad adulta, presentan asma o rinoconjuntivitis alérgica. El riesgo de un hijo de desarrollar DA, si uno de los progenitores la ha sufrido, es del $50 \%$, un porcentaje que sube al $80 \%$ si ambos progenitores padecen la enfermedad $(1,2,5)$.

Es la enfermedad dermatológica más frecuente en la infancia. Su incidencia ha ido aumentando a lo largo de los años, y actualmente se considera que afecta entre un 10 y un $30 \%$ de la población infantil, como lo revelan diversos estudios que se han realizado en España en los últimos años.

El estudio ELIHO analizó los factores de riesgo y prevalencia familiar de la dermatitis atópica en España. Se realizó en consultas pediátricas entre noviembre del 2003 y marzo del 2004. Como conclusión se encontró, entre los pacientes con dermatitis atópica, una clara predisposición genética (6).

Otros estudios han servido para conocer la prevalencia de la patología, como el estudio sobre prevalencia de la dermatitis atópica en escolares de Granada, de Guiote-Domínguez et al. (7) en 2008 para conocer la prevalencia de la patología en una población escolar.

La prevalencia y la incidencia de la patología van en aumento y el farmacéutico comunitario puede y debe realizar una labor esencial como educador sanitario para los cuidadores de estos pacientes, para de esta manera ayudar a garantizar una buena calidad de vida al paciente y contribuir al mejor control de los brotes.

En el ámbito de la farmacia comunitaria no se ha realizado ningún estudio que analice los diferentes aspectos de la dermatitis atópica, de cómo el farmacéutico comunitario puede abordar a los pacientes, y cómo y cuándo puede actuar o bien derivar al dermatólogo. Es por ello que desde la Sociedad Española de Farmacia Comunitaria (SEFAC) se proyectó el estudio DAFAC (Dermatitis Atópica en Farmacia Comunitaria).

Ante el desconocimiento por buena parte de la población, tanto de la enfermedad como de los cuidados que requiere la piel afectada, y la necesidad de disponer en la farmacia comunitaria de un protocolo de actuación, para que el farmacéutico pudiera abordar las consultas que recibía sobre la patología y acordar con los médicos implicados unos criterios consensuados de derivación, en el año 2009 el grupo de Dermatología de SEFAC, conjuntamente con un grupo de especialistas del departamento de Dermatología del Hospital del Mar de Barcelona y con el patrocinio de laboratorios LETI, se planteó la elaboración de un procedimiento de actuación.

Se realizó una prueba piloto con una muestra de farmacias comunitarias, cuyos resultados fueron presentados en el IV Congreso Nacional de Farmacéuticos Comunitarios: Estudio piloto para evaluar el protocolo de actuación en las consultas por dermatitis atópica en la farmacia comunitaria (8). Las conclusiones de dicho piloto derivaron en el planteamiento de un estudio a mayor escala, que aquí se presenta: Estudio DAFAC 2011, con el fin de evaluar su utilidad.

\section{Objetivos}

Objetivo general

- Evaluar la utilidad de un protocolo de actuación del farmacéutico ante las consultas realizadas sobre dermatitis atópica en la farmacia comunitaria.

\section{Objetivos específicos}

- Diseñar conjuntamente con el colectivo médico un protocolo de actuación ante las consultas recibidas en la farmacia sobre lesiones cutáneas sospechosas de dermatitis atópica.

- Acordar conjuntamente los criterios de derivación.

- Conocer cuáles son los motivos de consulta más habituales.

- Determinar en qué casos constituyen motivo de derivación al médico, o pueden ser abordadas por el farmacéutico comunitario, con la formación adecuada y siguiendo el protocolo de actuación.

- Analizar el grado de conocimiento del cuidado adecuado de la piel del paciente atópico por parte de los cuidadores del mismo.

\section{Método \\ Diseño}

Estudio epidemiológico para evaluar la demanda que el farmacéutico comunitario recibe sobre lesiones cutáneas sospechosas de dermatitis atópica y analizar si los pacientes o sus cuidadores son conocedores de los cuidados que requiere la piel atópica.

\section{Ámbito}

Farmacias comunitarias de todo el ámbito estatal español. El estudio se realizó entre los meses de febrero del 2011 y enero del 2012.

\section{Sujetos}

Criterios de inclusión: pacientes de cualquier edad (aunque la prevalencia es mayor en menores de 14 años) que, por sí mismos o mediante un familiar o cuidador, consultan un problema de salud dermatológico, de forma que el farmacéutico comunitario siguiendo el protocolo de actuación pueda determinar si es una lesión sospechosa de dermatitis atópica o no.

Criterios de exclusión: lesiones que no cumplen los criterios mayores de dermatitis atópica (2)

\section{Diseño del protocolo \\ de actuación}

Se realizaron reuniones de los farmacéuticos comunitarios del grupo de Dermatología de SEFAC con los médicos del Departamento de Dermatología del Hospital del Mar (Barcelona). Se consensuó un procedimiento de actuación en el que se establecían los criterios de derivación al médico especialista y se evaluaba la necesidad 


\section{Edad del paciente}
a) $0-2$ años
b) 2-6 años
c) 6-14 años
d) $>14$ años

2. Sexo del paciente
a) Masculino
b) Femenino

3. Zona de residencia
a) Urbana ( $>10.000$ habitantes)
b) Suburbana: (2.000-10.000 habitantes)
c) Rural (<2.000 habitantes)

4. Tras aplicar el protocolo, ¿existen criterios de derivación al dermatólogo?
a) Si (Indicar los criterios de derivación detectados)
b) No

5. El paciente, ¿ha sido diagnosticado previamente de dermatitis atópica?
a) $\mathrm{Si}$
b) No

6. Motivo de la consulta (marcar todos los necesarios)
a) Prurito
b) Irritación
c) Sequedad cutánea
d) Brote de dermatitis atópica
e) Sobreinfección de las lesiones
f) Dificultad para dormir, ansiedad, estrés
g) Demanda de información de dermatitis atópica

7. Si existe diagnóstico previo de DA, ¿utiliza productos adecuados para el cuidado de la piel atópica?

a) Si, de higiene y emoliencia

b) Si, pero solo de higiene

c) Sí, pero solo de emoliencia

d) Si, el tratamiento farmacológico prescrito por el medico

e) Si, el tratamiento farmacológico prescrito por el médico y productos emolientes

f) $\mathrm{Si}$, el tratamiento farmacológico prescrito y productos de higiene y emoliencia

g) No, ni de higiene ni de emoliencia

8. En caso de que en la pregunta anterior el farmacéutico identifique un mal cumplimiento en referencia al uso de productos adecuados para la piel atópica, indique el motivo que señalan los cuidadores

a) Por el elevado coste de los productos

b) Porque no creen que sean ni eficaces ni necesarios

c) Porque el/la niño/a es reacio/a a tener que aplicarse los productos cada día

d) Porque desconocian la importancia del cuidado diario para la mejora de la calidad de vida

9. Si utiliza productos para la piel atópica, ¿cuándo los utiliza?
a) Tratamiento farmacológico solo durante el brote
b) Tratamiento farmacológico en el brote y entre brotes
c) Tratamiento farmacológico y emoliencia en el brote
d) Tratamiento farmacológico durante el brote y emolientes entre brotes
e) Solamente emoliencia en el brote
f) Solamente emoliencia durante el brote y entre brotes
g) Otros. Especificar

10. Indicación o acción farmacéutica
a) Información sobre el problema de salud
b) Tratamiento farmacológico
c) Higiene y emoliencia
d) Coadyuvante
e) Fototerapia/Homeopatía
f) Otros

11. En relación a la sobreinfección de las lesiones, ¿consideran los cuidadores del niño atópico que se le infectan las lesiones?

a) No, nunca

b) Solo ocasionalmente

c) Si, siempre que tiene un brote de la enfermedad de derivar al paciente a la consulta del médico o bien la posibilidad de tratar al paciente en la farmacia y las herramientas terapéuticas, farmacológicas o no que el farmacéutico utilizaría para la resolución o alivio del problema de salud planteado (9).

Este procedimiento fue pilotado por 40 farmacéuticos para comprobar su aplicabilidad. Posteriormente se propuso participar en el estudio a los farmacéuticos comunitarios socios de SEFAC. En una primera fase se impartió la formación online a través de la plataforma Letiderma y posteriormente se realizó el registro de las consultas recibidas en las farmacias comunitarias entre febrero del 2011 y enero del 2012.

\section{Formación de los farmacéuticos} participantes

Patrocinada por Laboratorios LETI ${ }^{\circledR}$, se creó una plataforma (Letiderma), a través de la cual se realizó una formación online con vídeos y material teórico acreditada con 4,3 créditos. Al finalizar el curso y tras evaluación de su aprovechamiento, los farmacéuticos acreditados registraron los casos que recibieron en la farmacia comunitaria y la actuación que realizaban contestando a un cuestionario (figura 1).

\section{Procedimiento}

En el estudio DAFAC 2011 la captación de sujetos participantes en el estudio no está sujeta a variables, es decir se realiza registro de todas las consultas recibidas en la farmacia sobre lesiones dermatológicas susceptibles de ser indicativas de patología de dermatitis atópica.

Tras la identificación por parte del farmacéutico se realiza el cuestionario y la recogida de datos.

\section{Expresión y análisis}

de los resultados

El análisis estadístico se ha realizado utilizando el software de estadística SPSS 19.0 para Windows. En todas las tablas se describe el número de casos válidos (no se describen los valores perdidos).

Para el análisis estadístico se han creado 4 variables:

Cumplimiento (malo vs bueno): los casos en que el farmacéutico ha

Figura 1 Cuestionario de recogida de datos DAFAC 2011 


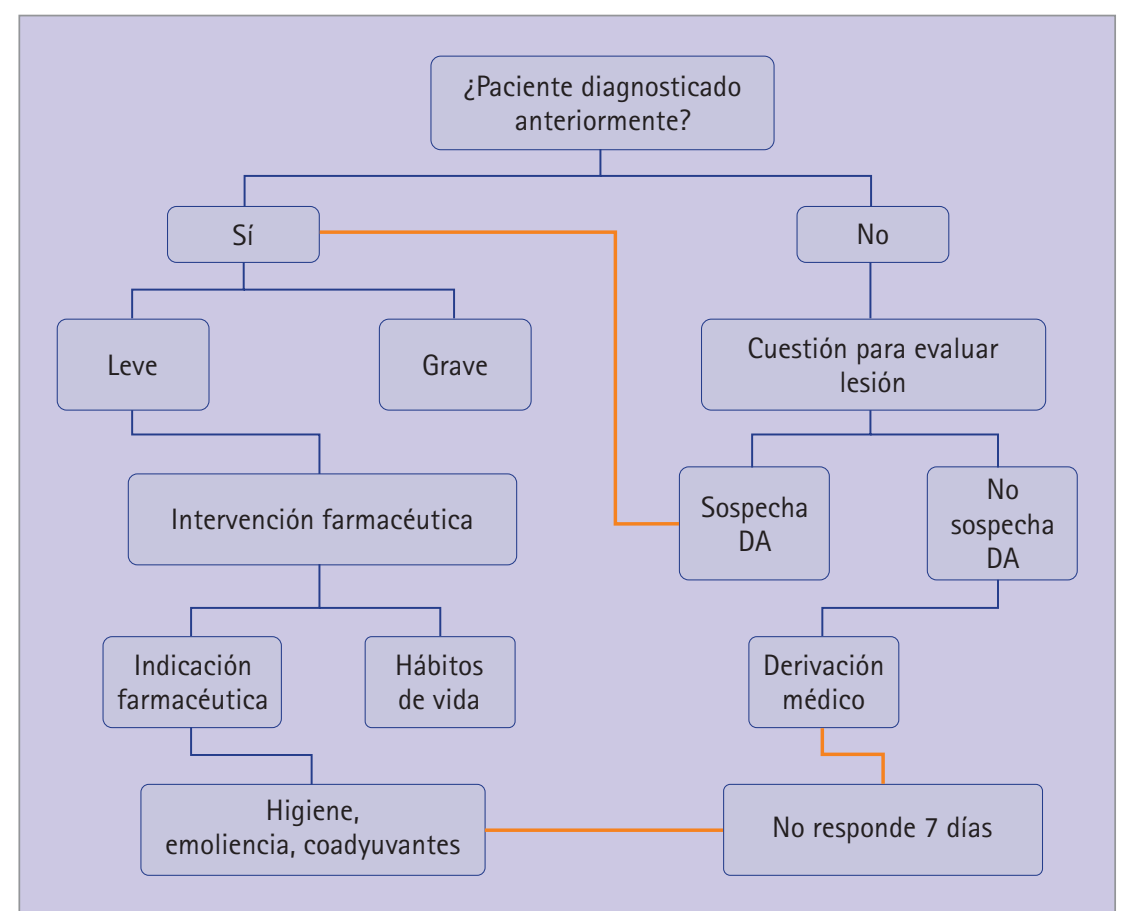

Figura 2 Protocolo de actuación y criterios de derivación

detectado un mal cumplimiento y ha descrito los motivos, se han considerado como casos "mal cumplimiento", el resto se han considerado como "buen cumplimiento".

Motivos de consulta: a partir de los diversos motivos de consulta descritos se han creado 2 variables de agrupación:

- 1 motivo vs. $>2$ motivos

- 1 motivo vs. 2-3 motivos vs. $>3$ motivos

Sobreinfección (no vs. sí): para describir la sobreinfección, en función de las respuestas a la pregunta “ ¿consideran los cuidadores del niño atópico que se le infectan las lesiones?", se han agrupado las categorías "no nunca" versus "solo ocasionalmente" y "si, siempre que tiene un brote de la enfermedad".

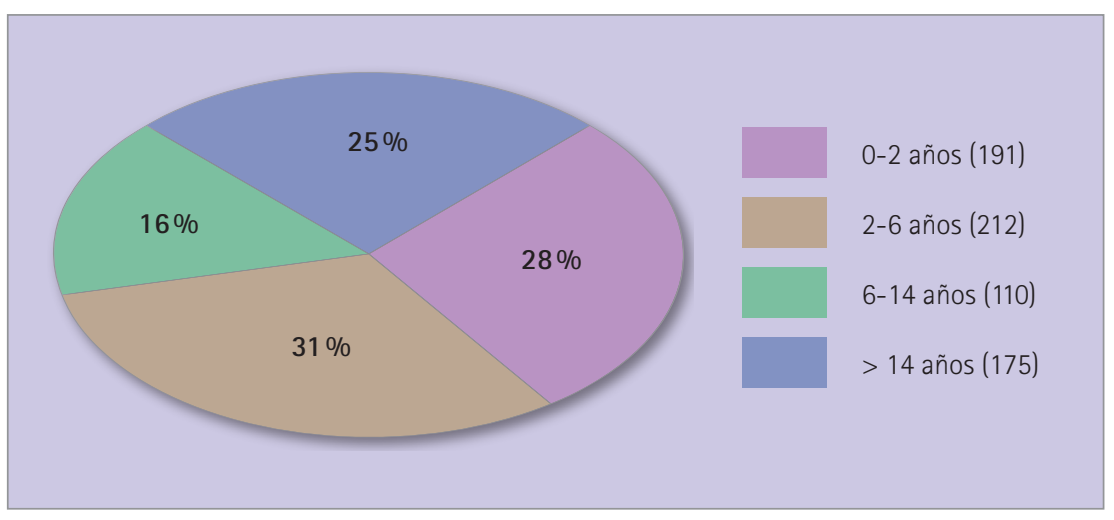

Figura 3 Distribución etaria de los pacientes
- No respuesta al tratamiento: si el paciente ya tiene instaurado un tratamiento para la dermatitis y no mejora su sintomatología, será necesaria la derivación al médico.

- Exacerbación de las lesiones (zonas crítica, blefaritis): la derivación al médico también debe considerarse si las lesiones afectan zonas críticas (cara, parpados, etc.)

- Sospecha de sobreinfección (costras, exudación abundante, fiebre, etc.).

- Varicela: si el paciente presenta brotes de atopía conjuntamente con la varicela, esto supone un criterio de gravedad de derivación urgente al médico.

En el estudio DAFAC participaron 259 farmacéuticos de 15 comunidades autónomas, que registraron un total de 688 intervenciones ante consultas por lesiones dermatológicas sospechosas de DA.

La distribución etaria de los pacientes se refleja en la figura 3 . Se ha observado que existe una mayor incidencia de la patología en los menores de 14 años. La distribución por sexo corresponde a 354 (51,5\%) de sexo masculino y 334 (48,5\%) del femenino, no existiendo diferencia significativa en la variable sexo del paciente.

Los motivos de consulta se reflejan en la figura 4. Tras aplicar el protocolo de actuación en la farmacia comunitaria, se advierte que en el $70 \%$ de los casos registrados no se identificaron criterios de derivación al dermatólogo. En estos casos se aplicó el protocolo de actuación para realizar una indicación farmacéutica y/o indicación de hábitos higienicodietéticos adecuados.

Se ha observado una relación significativa entre varios motivos de consulta y la existencia de criterios de derivación al dermatólogo: prurito, irritación, brote agudo de DA, dificultad para dormir, ansiedad y estrés, y sobreinfección. En todos los casos denota una mayor gravedad de los pacientes que requieren ser derivados al dermatólogo (tabla 1).

La sobreinfección, como motivo de consulta, es la que muestra una relación estadística más intensa con la necesidad de derivar al dermatólogo, ya que más del $80 \%$ de los pacientes con sobreinfección cumplen los criterios de derivación. Esta relación 


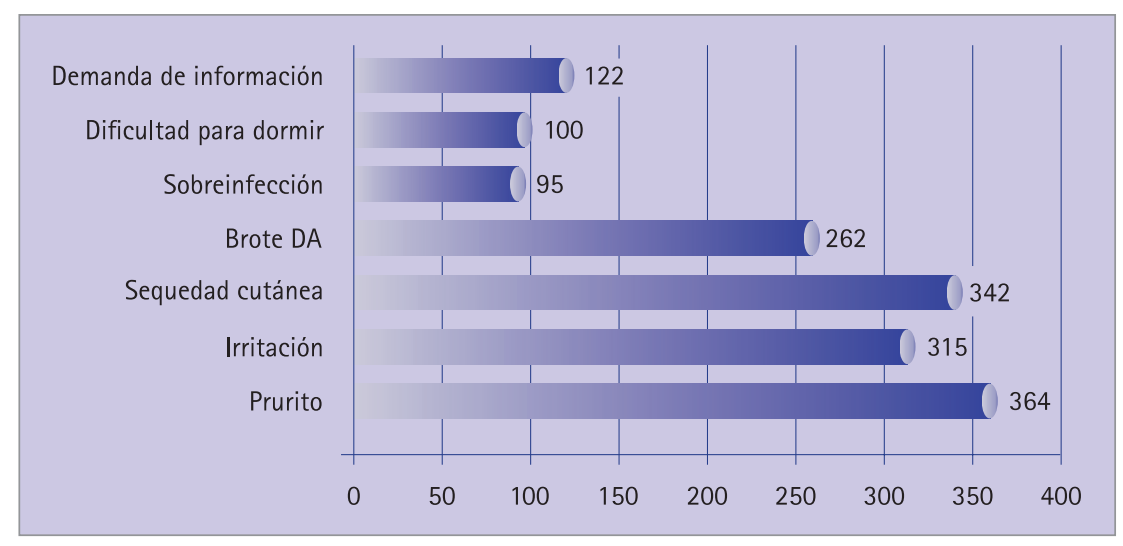

Figura 4 Motivos de consulta

Tabla 1 Criterios de derivación al dermatólogo tras aplicar el protocolo

\begin{tabular}{|c|c|c|c|c|c|c|}
\hline \multirow{3}{*}{ Factores } & & \multicolumn{4}{|c|}{ Criterios de derivación al dermatólogo } & \multirow[b]{3}{*}{ Significación* } \\
\hline & & \multicolumn{2}{|r|}{ Si } & \multicolumn{2}{|c|}{ No } & \\
\hline & & $n$ & $\%$ & $\mathrm{n}$ & $\%$ & \\
\hline \multirow{2}{*}{$\begin{array}{l}\text { Prurito (motivo } \\
\text { de consulta) }\end{array}$} & No & 67 & $20,70 \%$ & 257 & $79,30 \%$ & \multirow{2}{*}{$p<0,001$} \\
\hline & Si & 140 & $38,50 \%$ & 224 & $61,50 \%$ & \\
\hline \multirow{2}{*}{$\begin{array}{l}\text { Irritación (motivo } \\
\text { de consulta) }\end{array}$} & No & 83 & $22,30 \%$ & 290 & $77,70 \%$ & \multirow{2}{*}{$p<0,001$} \\
\hline & Si & 124 & $39,40 \%$ & 191 & $60,60 \%$ & \\
\hline \multirow{2}{*}{ Brote DA } & No & 98 & $23,00 \%$ & 328 & $77,00 \%$ & \multirow{2}{*}{$p<0,001$} \\
\hline & Si & 109 & $41,60 \%$ & 153 & $58,40 \%$ & \\
\hline \multirow{2}{*}{$\begin{array}{l}\text { Sobreinfección } \\
\text { (motivo de } \\
\text { consulta) }\end{array}$} & No & 130 & $21,90 \%$ & 463 & $78,10 \%$ & \multirow[b]{2}{*}{$p<0,001$} \\
\hline & Si & 77 & $81,10 \%$ & 18 & $18,90 \%$ & \\
\hline \multirow{2}{*}{$\begin{array}{l}\text { Alteración sueño/ } \\
\text { ansiedad/estrés } \\
\text { (motivo de consulta) }\end{array}$} & No & 144 & $24,50 \%$ & 444 & $75,50 \%$ & \multirow[b]{2}{*}{$p<0,001$} \\
\hline & Si & 63 & $63,00 \%$ & 37 & $37,00 \%$ & \\
\hline \multirow{4}{*}{$\begin{array}{l}\text { En relación a la } \\
\text { sobreinfección } \\
\text { de las lesiones, } \\
\text { ¿consideran los } \\
\text { cuidadores del } \\
\text { niño atópico que } \\
\text { se le infectan las } \\
\text { lesiones? }\end{array}$} & No, nunca & 71 & $20,20 \%$ & 281 & $79,80 \%$ & \multirow{4}{*}{$p<0,001$} \\
\hline & $\begin{array}{l}\text { Solo } \\
\text { ocasionalmente }\end{array}$ & 98 & $40,80 \%$ & 142 & $59,20 \%$ & \\
\hline & $\begin{array}{l}\text { Si, siempre que } \\
\text { tiene un brote }\end{array}$ & 26 & $63,40 \%$ & 15 & $36,60 \%$ & \\
\hline & Total & 195 & $30,80 \%$ & 438 & $69,20 \%$ & \\
\hline \multirow{3}{*}{$\begin{array}{l}\text { Antecedentes de } \\
\text { sobreinfección }\end{array}$} & No & 71 & $20,20 \%$ & 281 & $79,80 \%$ & \multirow{3}{*}{$p<0,001$} \\
\hline & Si & 124 & $44,10 \%$ & 157 & $55,90 \%$ & \\
\hline & Total & 195 & $30,80 \%$ & 438 & $69,20 \%$ & \\
\hline
\end{tabular}

* Prueba chi cuadrado de Pearson.

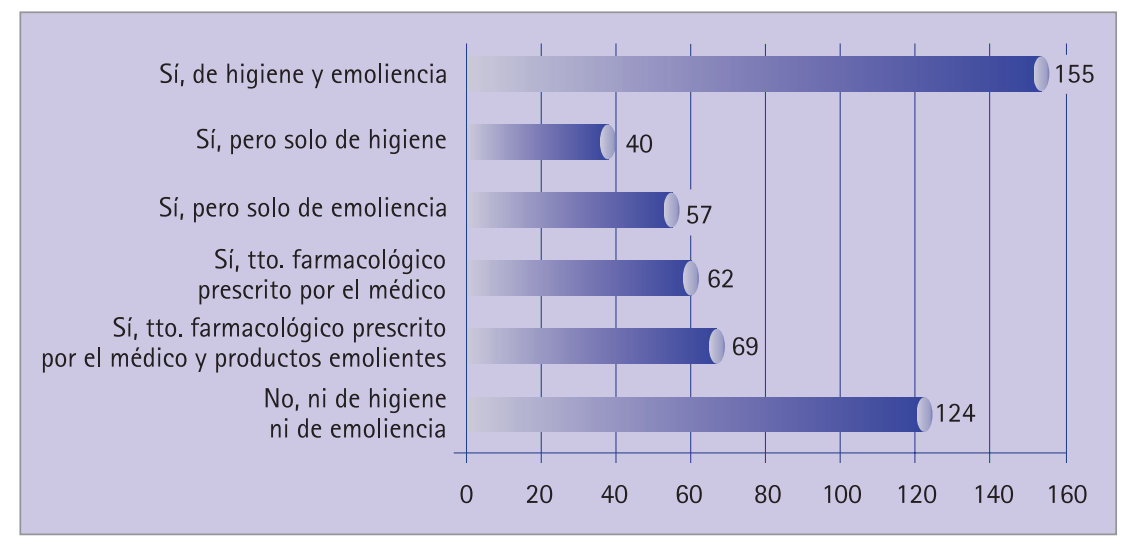

Figura 5 ¿Utiliza productos adecuados para el cuidado de la piel atópica? es igualmente intensa cuando la sobreinfección de las lesiones, independientemente o no de ser un motivo de consulta, forma parte del historial clínico del paciente.

El uso de productos para el cuidado de la piel atópica se muestra en la figura 5. Se ha observado que solo un $25 \%$ de los pacientes que hicieron las consultas utilizaba productos adecuados de higiene y emoliencia. Se encontró una relación significativa $p=0,011$ entre la edad de los pacientes y el cumplimiento de las medidas terapéuticas, de forma que este es mayor cuando se trata de niños muy pequeños y el cumplimiento corresponde al de sus padres. A lo largo de toda la infancia, el cumplimiento disminuye, siendo mínimo entre los 6 y los 14 años y volviendo a aumentar a partir de los 14 años. La zona de residencia también parece afectar significativamente al cumplimiento, de modo que este es más bajo en las zonas rurales $(p=0,014)$ (tabla 2).

Los identificados como mal cumplidores del uso de productos adecuados refirieron causas como el desconocimiento de los cuidados necesarios en un $44 \%$ o el alto coste de los productos en un $27 \%$ (figura 6).

El 35\% de los pacientes refiere sobreinfección de heridas en alguna ocasión. Se ha observado que dos motivos de consulta, el brote activo de DA y la dificultad para dormir, ansiedad y estrés, se relacionan con mayor frecuencia con un historial de sobreinfección de las lesiones; mientras que, cuando el motivo de consulta es la sequedad cutánea, la proporción de pacientes con historial de sobreinfección es significativamente menor.

Se ha observado una relación significativa entre la sobreinfección de las lesiones y la edad, de forma que la mayor proporción de pacientes con sobreinfección se halla entre los mayores de 14 años. No obstante, no puede descartarse que se trate de una relación esperable al haber más tiempo de evolución de la enfermedad.

En este sentido, también se observa una relación significativa entre la existencia de un diagnóstico previo de DA y el historial de sobreinfección de las lesiones.

Se destaca como resultado del estudio que en el $70 \%$ de las consultas recibidas no existían criterios de derivación al dermatólogo y por tanto han sido consultas resueltas 
Tabla 2 Relación entre el cumplimiento y la edad del paciente y su zona de residencia

\begin{tabular}{|c|c|c|c|c|c|c|}
\hline & & \multicolumn{4}{|c|}{ Cumplimiento } & \multirow[b]{3}{*}{ Significación* } \\
\hline & & & lo & & eno & \\
\hline \multicolumn{2}{|l|}{ Factores } & $n$ & $\%$ & $n$ & $\%$ & \\
\hline \multirow{4}{*}{ Edad del paciente } & $0-2$ años & 94 & $49,20 \%$ & 97 & $50,80 \%$ & \multirow{4}{*}{$p<0,001$} \\
\hline & 2-6 años & 131 & $61,80 \%$ & 81 & $38,20 \%$ & \\
\hline & 6-14 años & 74 & $67,30 \%$ & 36 & $32,70 \%$ & \\
\hline & > 14 años & 103 & $58,90 \%$ & 72 & $41,10 \%$ & \\
\hline \multirow{3}{*}{$\begin{array}{l}\text { Zona de } \\
\text { residencia }\end{array}$} & Urbana & 241 & $57,70 \%$ & 177 & $42,30 \%$ & \multirow{3}{*}{$p<0,001$} \\
\hline & Suburbana & 87 & $52,70 \%$ & 78 & $47,30 \%$ & \\
\hline & Rural & 74 & $70,50 \%$ & 31 & $29,50 \%$ & \\
\hline
\end{tabular}

* Prueba chi cuadrado de Pearson.

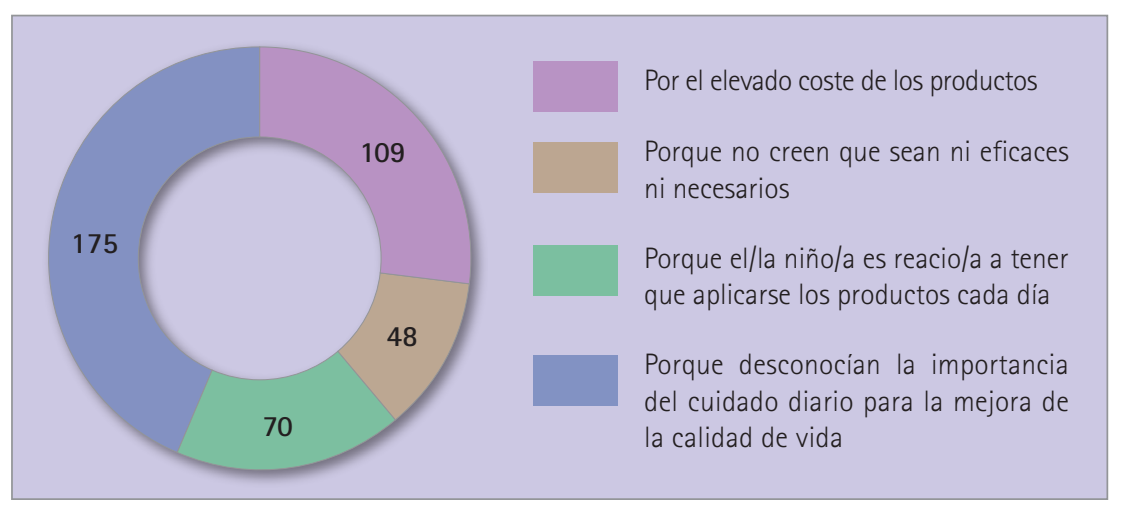

Figura 6 Causas del mal cumplimiento

por el profesional farmacéutico, por lo que el rol de detección y recomendación farmacéutica es muy importante.

En más de la mitad de las consultas se ha detectado un mal cuidado de la piel atópica, identificándose una infrautilización de los productos para tratar la piel atópica (9). Este último dato refleja en primer lugar el papel de educador sanitario del farmacéutico y en segundo lugar la falta de información que reciben los pacientes atópicos.

La sobreinfección de lesiones, complicación de la DA con graves consecuencias, presente en un 35\% de los casos, puede atenuarse si los productos de higiene utilizados diariamente contienen ingredientes que puedan favorecer la flora sa- de la calidad de vida del paciente. De este modo, siguiendo los criterios de actuación y derivación consensuados con los dermatólogos del Hospital del Mar de Barcelona, el farmacéutico comunitario ejerce una importante labor profesional, actuando además como educador sanitario en colaboración con el profesional médico.

\section{Referencias bibliográficas}

1. Gascó A, Buesa S, Labat MP, Lujan T, Zaborros E, Higueras MI [editores]. Dermatitis Atópica [monografía en CD-ROM]. Zaragoza: Colegio Oficial de Farmacéuticos de Zaragoza; 2004.

2. Guerra Tapia A, González-Guerra E. Dermatitis atópica [monografía en Internet]. Actualizaciones Aula de la Farmacia. 2001. [acceso 12/6/13]. Disponible en http://www.auladelafarmacia. org/Documentos/doc/dermatitis.pdf

3. Herrero JE, Moreno F. Protocolo de dermatitis atópica. Farmacéuticos Comunitarios. 2010;2(3):116-21.

4. Santamaría LF. Las células de Langerhans en la inmunidad cutánea, con especial referencia a la dermatitis atópica. Act Dermatolog. 1998;3:179-81.

5. Rudikoff D, Lebwohl M. Atopic dermatitis. The Lancet. 1998;351:1715-21. doi:10.1016/S0140-6736(97)12082-7

6. Blanco Quirós A, Díaz Castella JM, Balañá Vilanova M, Valveny Llobet N. Factores de riesgo y prevalencia familiar de la dermatitis atópica en España (estudio ELIHO). An Pediatr (Barc). 2005;63(6):480-8. doi:10.1016/S16954033(05)70246-0

7. Guiote-Domínguez MV, Muñoz-Hoyos A, Gutiérrez-Salmerón MT. Prevalencia de dermatitis atópica en escolares de Granada. Actas Dermosifiliogr. 2008;99(8):628-38. doi.org/10.1016/ S0001-7310(08)74759-8

8. Moreno Fernández P. Estudio piloto para evaluar el protocolo de actuación en las consultas por dermatitis atópica en la farmacia comunitaria. Farmacéuticos Comunitarios. 2010;2(Supl.1):S51-S52.

9. Fernández del Pozo MB, Carbajo Espejo JM, Palazón Ors JJ. Tratamiento cosmético en afecciones dermatológicas. En: Consejo General de Colegios Oficiales de Farmacéuticos. Atención Farmacéutica en Dermofarmacia. $1^{\text {a }} \mathrm{ed}$. Madrid: BGA Asesores; 2008. p. 398410. 